\title{
Morphological and spectroscopic studies on the vertically aligned zinc oxide nanorods grown on low and high temperature deposited seed layer
}

\author{
(C) A. Rayerfrancis ${ }^{1,2}$, Bhargav P. Balaji ${ }^{1,2, \uparrow}$, N. Ahmed ${ }^{1,2}$, C. Balaji ${ }^{2}$ \\ ${ }^{1}$ Department of Physics, SSN College of Engineering, \\ Kalavakkam, India \\ ${ }^{2}$ SSN Research Center, SSN College of Engineering, \\ Kalavakkam, India \\ ฯE-mail: balajibhargavp@ssn.edu.in
}

(Received 20.02.2017. Received after revision 4.04.2017)

\begin{abstract}
Vertically aligned zinc oxide nanorods were grown on low and high temperature deposited aluminium doped zinc oxide seed layer by hydrothermal method and annealed to improve crystallinity. The morphology of the seed layer and the grown nanorods were studied by field emission scanning electron microscopy characterization technique. The properties of the zinc oxide nanorods were analyzed using laser spectroscopic studies. Resonant Raman spectroscopy reveals the unique increase in the $A_{1}(\mathrm{LO})$ mode of vibration with increase in count. The luminescence property of the nanorods was studied with photoluminescence spectrometer. The vertically aligned zinc oxide nanorods show, the very high band edge emission in the ultraviolet region of the electromagnetic spectrum.
\end{abstract}

DOI: $10.21883 /$ FTP.2017.12.45186.8562

\section{Introduction}

Being a naturally available abundant material on earth, having the unique electrical and optical properties with large exiton binding energy $(60 \mathrm{meV})$ and large band gap $(3.4 \mathrm{eV})$, zinc oxide $(\mathrm{ZnO})$ finds a tremendous amount of opportunities in research and scientific applications [1]. ZnO nanostructures show unique properties as compared to its bulk form. These nanostructures exists in many forms, 0-dimensional quantum dots [2], 1-dimensional nanowires, nanorods, nanoribbons [3-5], 2-dimensional nanoplasts, nanodiscs [6,7] and 3-dimensional nanofoam, nanoflowers etc. $[8-10]$. Due to its tunable electrical and optical properties, various forms of $\mathrm{ZnO}$, both doped and undoped, are considered as a potential candidates in many electronic applications such as flat panel displays, light emitting diodes, solar cells, piezoelectric generators as well as in cosmetics and therapy [11-16]. As the optical properties of the $\mathrm{ZnO}$ nano structures strongly dependent on the size and shape, controlling them becomes more important [17]. The property of seed layer plays an important role in the structural, morphological properties of the $\mathrm{ZnO}$ nanostructure. So, in this present work two types of seed layers were used, room temperature sputter deposited seed layer and high temperature sputter deposited seed layer. On these two types of seed layers, hydrothermal reaction was carried out at two different concentrations for two different time periods to study the properties of the grown $\mathrm{ZnO}$ nanostructures.

\section{Experimental}

$\mathrm{ZnO}$ nanorods were grown by hydrothermal method using zinc nitrate hexahydrate $\left(\mathrm{Zn}\left(\mathrm{NO}_{2}\right)_{3} \cdot 6 \mathrm{H}_{2} \mathrm{O}\right)$ hexamethylenetetramine $\left(\mathrm{C}_{6} \mathrm{H}_{12} \mathrm{~N}_{4}\right)$. Both chemicals were purchased from Sigma Aldrich and were used directly without any further purification. Deionized (DI) water was used throughout the process. For seed layer deposition $\mathrm{ZnO}$ ceramic sputtering targets doped with $2 \mathrm{wt} \% \quad \mathrm{Al}_{2} \mathrm{O}_{3}$ $(5 \mathrm{~N}$ purity) were purchased from ITASCO, Korea.

\subsection{Seed Layer Growth}

Aluminium doped Zinc Oxide (AZO) seed layers were deposited on Eagle $\mathrm{Xg}$ (Corning) glass substrate using DC magnetron sputtering (12" MSPT, Hind High Vacuum, India) technique. The substrates were initially washed with soap solution and rinsed. Subsequently sonicated in DI water for $15 \mathrm{~min}$, followed by sonicating in acetone for another $15 \mathrm{~min}$. Finally, in the cleaning procedure, the substrates were cleaned in 2-proponal vapour. Before doing sputtering to ensure the oxygen and other contaminant free environment, the chamber was initially evacuated up to $5 \cdot 10^{-6}$ mbar. The optimized process parameters for the AZO seed layer deposition are maintained constant throughout the process. AZO was deposited on substrates at two different temperatures (room temperature, $25^{\circ} \mathrm{C}$ and high temperature $250^{\circ} \mathrm{C}$ ) to study the effect of seed layer in the growth and dispersion of the nanorods.

\subsection{Zinc Oxide Nanorod Growth}

$\mathrm{ZnO}$ nanorods were grown by hydrothermal method using $\mathrm{Zn}\left(\mathrm{NO}_{2}\right)_{3} \cdot 6 \mathrm{H}_{2} \mathrm{O}$ and $\mathrm{C}_{6} \mathrm{H}_{12} \mathrm{~N}_{4}$ in an equal molar concentration. Both precursors mixed well in DI water separately to obtain a clear solution and then transferred into a Teflon lined stainless steel autoclave. The autoclave was closed tightly and kept in a furnace at $100^{\circ} \mathrm{C}$ for a particular time period. To study the impact of seed on 


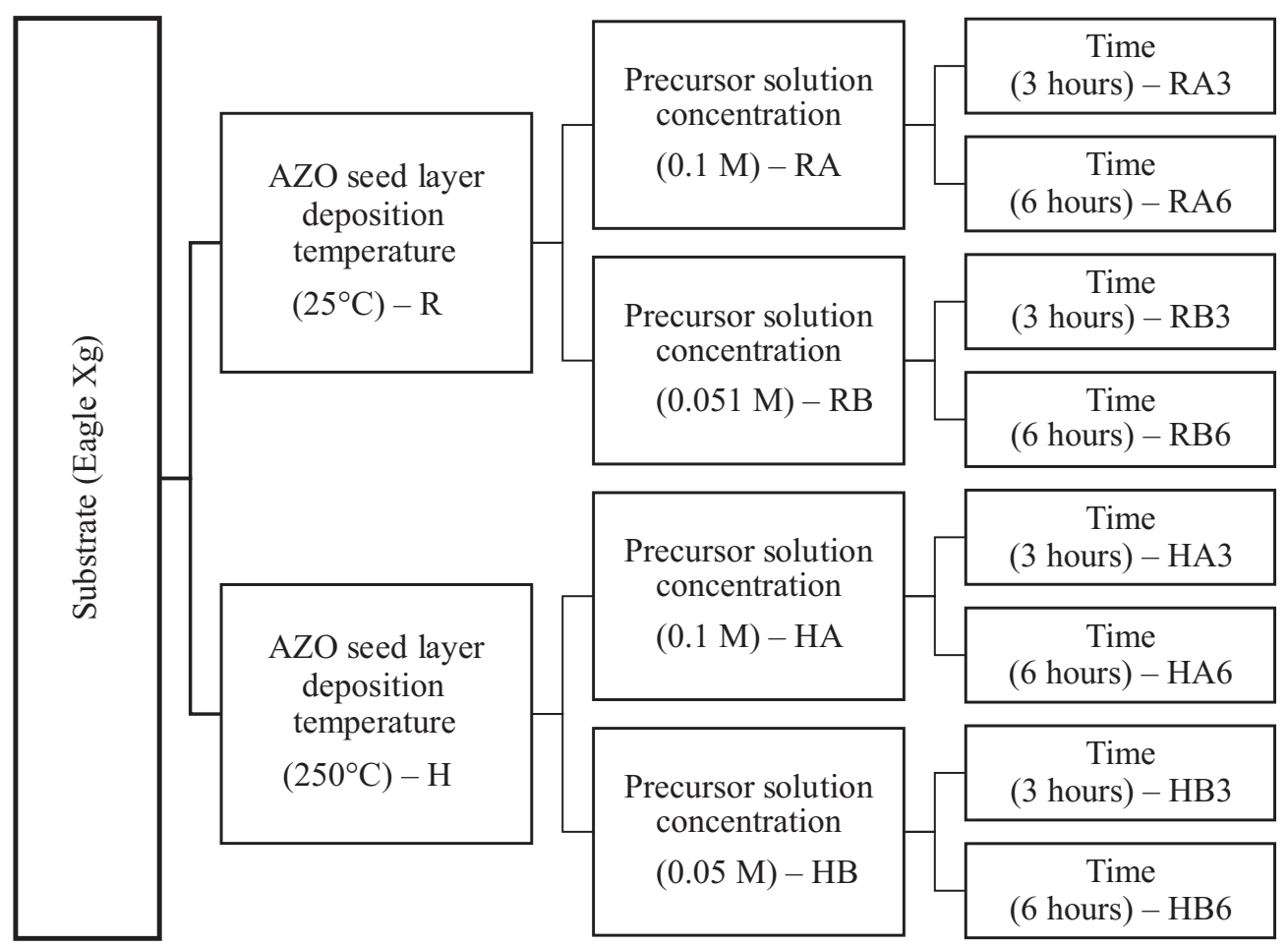

Figure 1. Experimental design of the hydrothermal reaction.

the growth of $\mathrm{ZnO}$ nanorods, seed layers were prepared at two different temperatures $\left(25^{\circ} \mathrm{C}\right.$ and $\left.250^{\circ} \mathrm{C}\right)$ on which the $\mathrm{ZnO}$ nanorods have been grown. On both seed layers, the solution concentration was varied, alike $0.1 \mathrm{M}$ and $0.05 \mathrm{M}$. Finally the hydrothermal reaction was carried out for $3 \mathrm{~h}$ and $6 \mathrm{~h}$ to study the growth of $\mathrm{ZnO}$ nanorods. The samples were annealed at $450^{\circ} \mathrm{C}$ before characterization to improve the crystalline and structural properties. The detailed chart on the growth of $\mathrm{ZnO}$ nanorods using various process parameters is given in the Fig. 1 . For understanding „ $\mathrm{R}^{\text {“ }}$ given to the seed layer deposited at room temperature $\left(25^{\circ} \mathrm{C}\right)$ and „ $\mathrm{H}^{\prime \prime}$ given to the seed layer deposited at high temperature $\left(250^{\circ} \mathrm{C}\right)$. „A $\mathrm{A}^{\text {“ }}$ is assigned to the precursor solution of concentration $0.1 \mathrm{M}$ and „ $\mathrm{B}^{\text {“ }}$ assigned to the precursor solution of concentration $0.05 \mathrm{M}$.

The morphology of the nanorods was studied by field emission scanning electron microscope (FESEM, Zeis GEMINI) and the structure was studied by $X$-ray diffraction (XRD Cu- $K_{\alpha}$, Righaku). Micro-Raman and photoluminescence (PL) spectra of the samples were recorded (Renishaw, in Via) using $325 \mathrm{~nm}$ with $2400 \mathrm{gr} / \mathrm{mm}$ grating excitations in the backscattering configuration and thermoelectric cooled CCD detector using a 100x objective.

\section{Results and discussion}

\subsection{Electron Microscope studies}

Fig. 2 shows the FESEM image of $\mathrm{ZnO}$ nanorods grown at room temperature deposited AZO seed layer and Fig. 3 shows the FESEM images of $\mathrm{ZnO}$ nanorods grown at high temperature deposited AZO seed layer. In each set of process condition, three images are given lower magnification, higher magnification and a tilted image to understand the nanorods growth and dimension. A general similarity observed in both the set of FESEM images (nanorods grown on low as well as high temperature deposited seed layers with respect to time) that with the decrease in the precursor solution concentration, the growth of nanorods are more dispersed. In all cases, nanorods grown using $0.1 \mathrm{M}$ concentration solution were packed denser and in some cases they got aggregated with one another. Nanorods grown using $0.05 \mathrm{M}$ concentration precursor solution, individual nanorods can be seen in the top view of the FESEM image. With the increase of the solution concentration, the dispersion of the nanorods decreased, this is because the increase in the precursor solution concentration causes the increase in the nanorod diameter reported by Polsongkram etal. [18]. In all cases hydrothermal reaction was carried out for 2 different time periods, 3 and $6 \mathrm{~h}$ respectively. $6 \mathrm{~h}$ grown nanorods are almost double amount longer than the rods gown for $3 \mathrm{~h}$. The major difference between nanorods grown at the room and higher temperature deposited seed layers is the dispersion. During sputter deposition, the film deposited at room temperature, the atoms and molecules are directly nucleated on the substrate and then grow into islands so that the $\mathrm{ZnO}$ molecules are more strongly bound to each other than the substrate. This is because the energy of the sputtered species in the plasma is higher than the surface energy of the substrate. Volmer-Weber kind of growth 

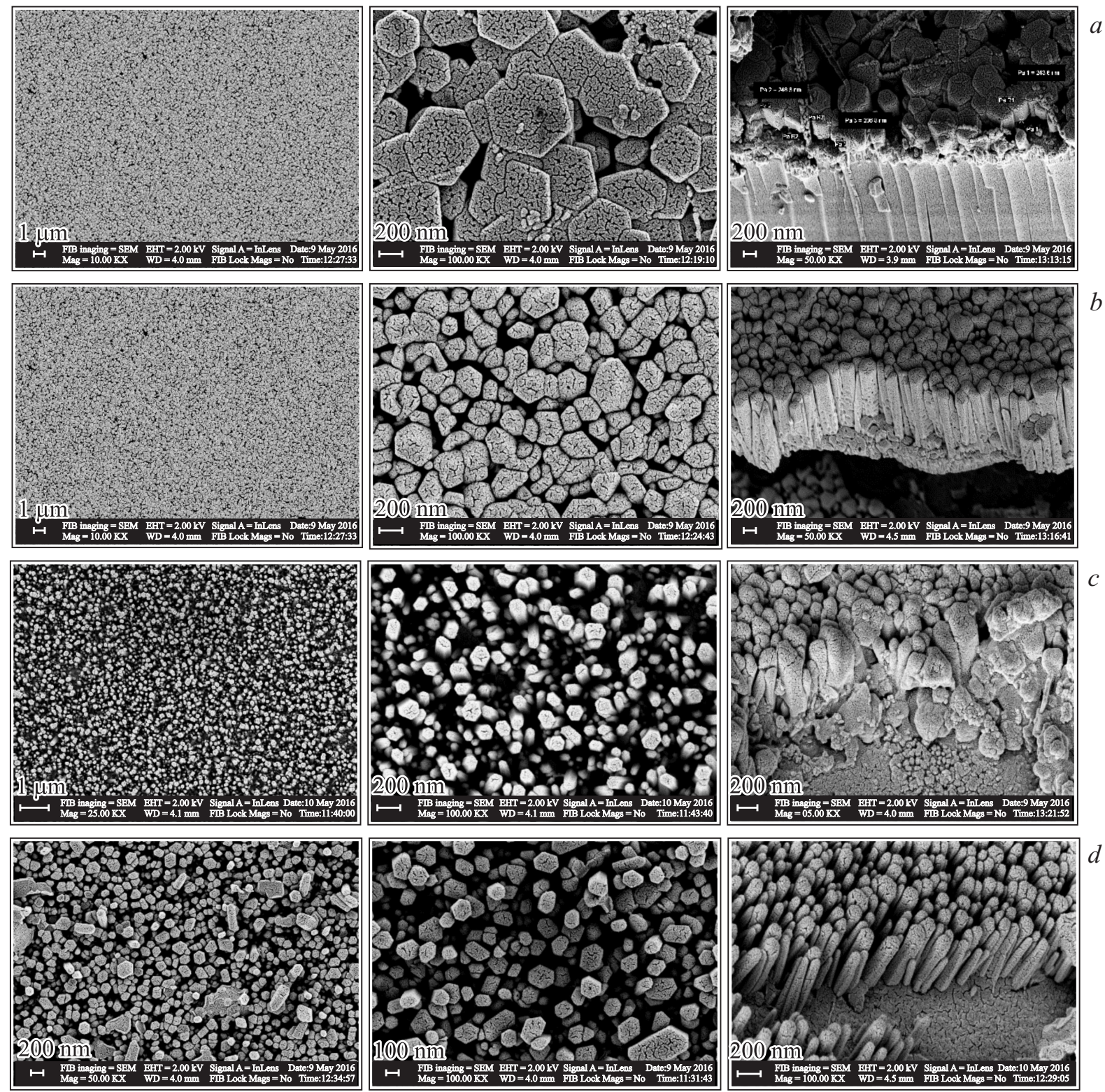

Figure 2. Electron microscope image of zinc oxide nanorods grown on low temperature deposited aluminium doped zinc oxide seed layer at (a) RA3, (b) RA6, (c) RB3 and (d) RB6.

takes place during this low temperature deposition and this results in an individual cluster kind of thin film as shown in Fig. 4, $a$. During high temperature deposition of seed layer, due to the high surface energy of the substrate, the bonding between the sputtered species in the plasma and the substrate is good enough to form layer plus island growth. This results in textured surface of the AZO film shown in Fig. 4, $b[19,20]$. This causes low temperature seeded nanorods grow in a more dispersed manner than the higher temperature seeded nanorods.

\section{2. $X$-ray Diffraction analysis}

The hydrothermally grown $\mathrm{ZnO}$ nanorods were subjected to annealing at $450^{\circ} \mathrm{C}$ for $2 \mathrm{~h}$ to improve the crystallinity.
Fig. 5 represents the $\mathrm{XRD}$ spectra of $3 \mathrm{~h}$ grown $\mathrm{ZnO}$ nanorods grown at $(a)$ room temperature deposited AZO seed and $(b)$ high temperature deposited AZO seed. Spectra shows only a peak at $34.52^{\circ}$, which corresponds to the (002) plane of the wurtzite structure of $\mathrm{ZnO}$ crystal (JCPDS \# 36-1451). This $c$-axis orientation of the nanorods confirms the crystallinity of the vertically grown nanorods.

\subsection{Raman spectroscopy studies}

Zinc oxide crystal possesses wurtzite structure and belongs to $C_{6 v}^{4} 4$ space group with two formula units in the primitive cell. Group theory designates that there are eight sets of optical phonon modes, $2 A_{1}, 2 B_{1}, 2 E_{1}$ and $2 E_{2}$ are present in $\mathrm{ZnO}$. The $A_{1}$ and $E_{1}$ modes are 

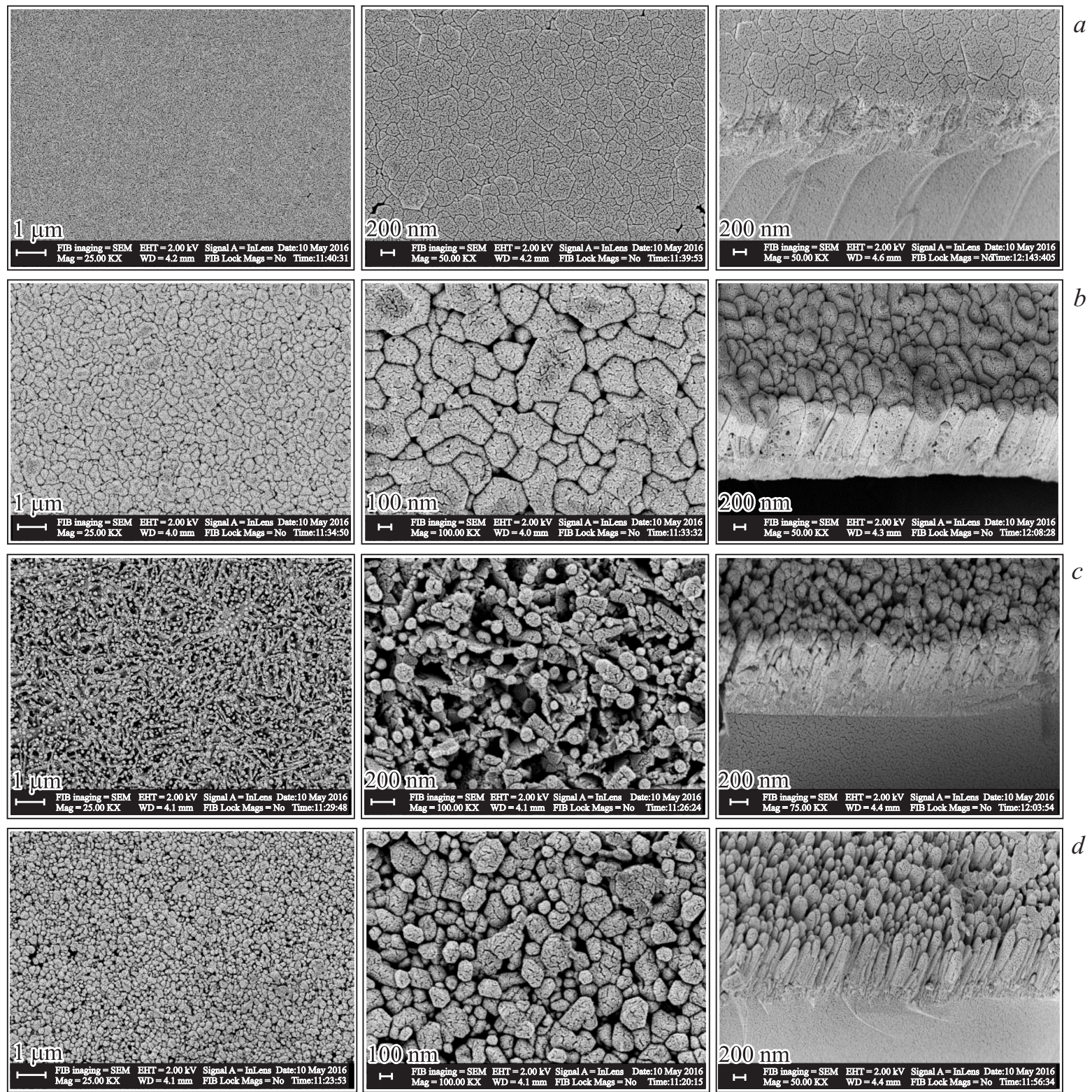

Figure 3. Electron microscope image of zinc oxide nanorods grown on low temperature deposited aluminium doped zinc oxide seed layer at $(a) \mathrm{HA} 3,(b) \mathrm{HA} 6,(c) \mathrm{HB} 3$ and $(d) \mathrm{HB} 6$.
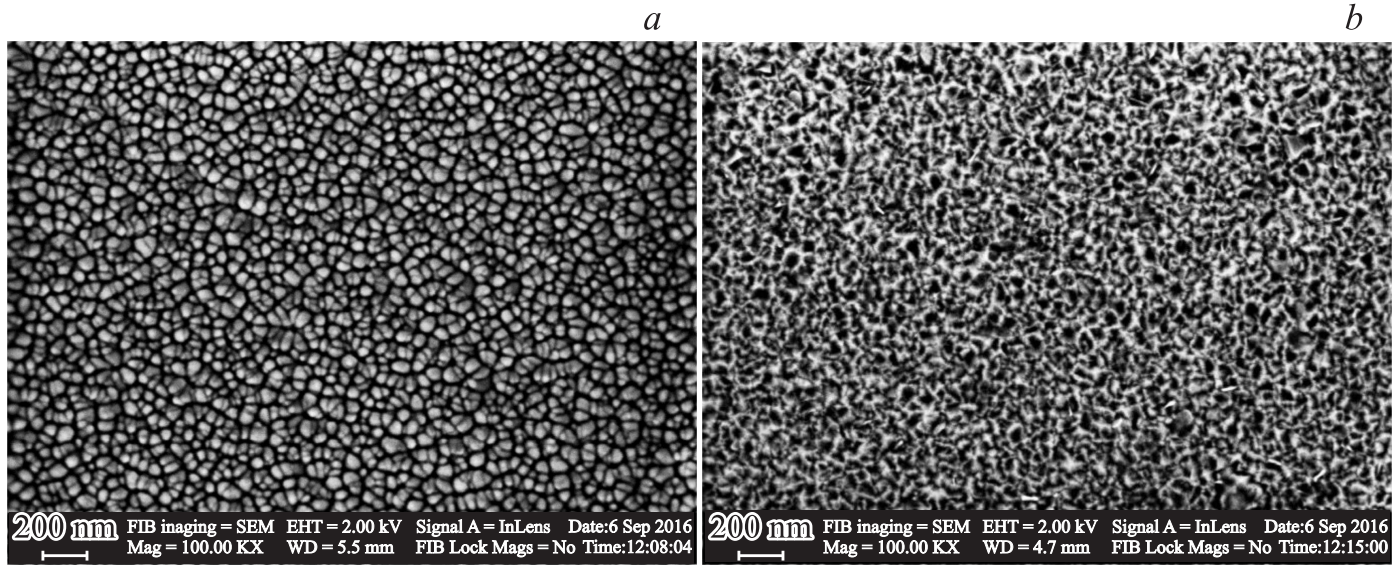

Figure 4. Electron microscope image of aluminium doped zinc oxide seed layer deposited at $(a)$ room temperature $\left(25^{\circ} \mathrm{C}\right)$ and $(b)$ high temperature $\left(250^{\circ} \mathrm{C}\right)$. 

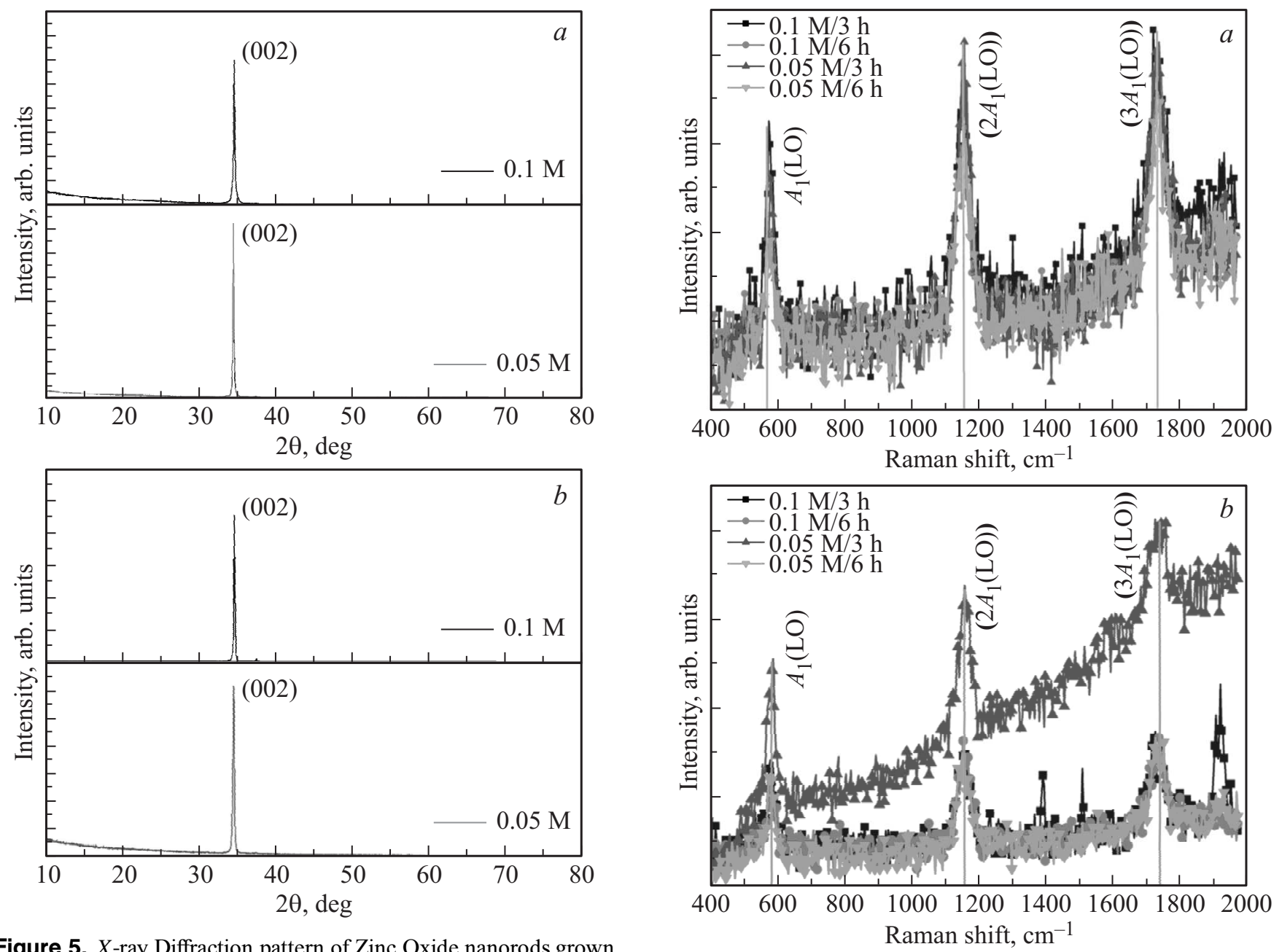

Figure 5. $X$-ray Diffraction pattern of Zinc Oxide nanorods grown on $(a)$ room temperature deposited Aluminium doped Zinc Oxide seed (RA3, RB3) and (b) high temperature deposited AZO seed (HA3, HB3).

polar and active with both Raman and infrared. The other two $E_{2}$ modes $\left(E_{2}\right.$ low, $E_{2}$ high $)$ are nonpolar, only Raman active. The vibrations of polar $A_{1}$ and $E_{1}$ modes can polarize the unit cell, which creates a long range electrostatic field splitting the polar modes into longitudinal optical (LO) and transverse optical (TO) components [21]. In non-resonant Raman scattering with the energy of the incident photon below the optical gap of a semiconductor, the observable Raman modes are determined by the Raman selection rules considering the crystal symmetry as well as the propagation and polarization directions of the incident and scattered light, while resonant Raman scattering occurs when the energy of the incident or scattered photon approaches the optical gap of the material [22]. The resonant Raman spectra of annealed $\mathrm{ZnO}$ nanostructures grown using various process parameters were studied using $325 \mathrm{~nm}$ line of $\mathrm{He}-\mathrm{Cd}$-laser as excitation source. The excitation energy is close to the electronic interband transition energy of wurtzite $\mathrm{ZnO}$ [23]. From the Figs. 6, $a$ and $b$ the intense resonant multiphonon scattering is observed in the Raman

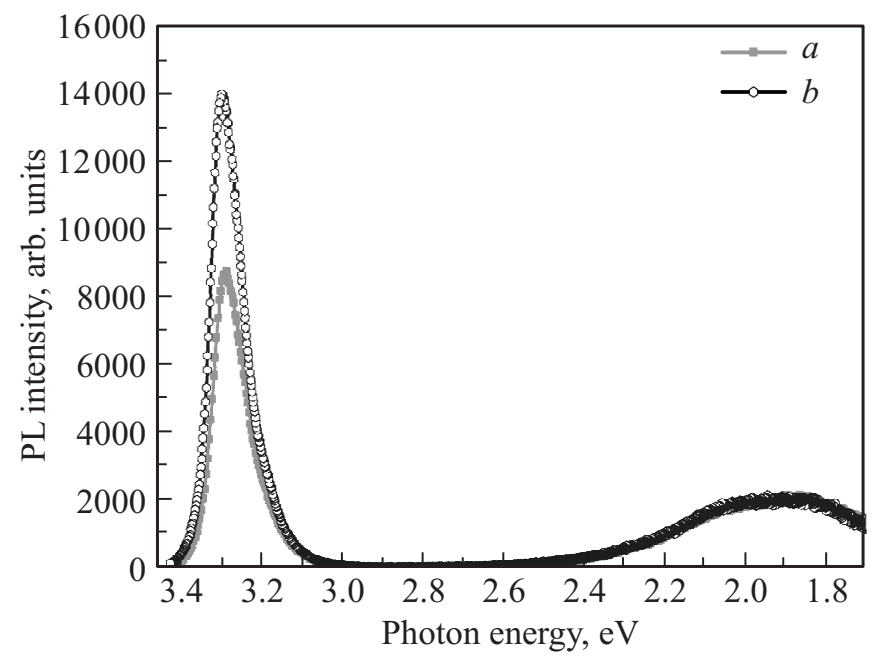

Figure 7. Photoluminescence image of vertically aligned Zinc oxide nanorods grown on $(a)$ low temperature deposited seed [RB6] and (b) high temperature deposited seed [HB3]. 
Spectra of the $\mathrm{ZnO}$ nanorod arrays. This multiphonon scattering is clearly visible up to three orders which are composed of three major bands centered at 570, 1154 and $1730 \mathrm{~cm}^{-1}$ and are identified as the first order $\left(A_{1}-\mathrm{LO}\right)$, the second order $\left(A_{2}-\mathrm{LO}\right)$ and the third order $\left(A_{3}-\mathrm{LO}\right)$ optical phonon peaks respectively. This is because of the excitation energy produced by the laser $(3.814 \mathrm{eV})$ is close to the photon scattering energy of $A_{1}-\mathrm{LO}$ phonons and thus the photon scattering by $A_{1}-\mathrm{LO}$ phonons is maximum enhanced [24]. According to the Raman selection rule, the peak at $570 \mathrm{~cm}^{-1}$ corresponds to the $A_{1}-\mathrm{LO}$ phonon, this will appear when $\mathrm{ZnO}$ nanorods grown along the $c$-axis [25]. Overtone modes up to three orders are observed. With an increase in the order of overtone, the count is also increased which is a sign of good crystallinity [26].

\subsection{Photoluminescence}

Photoluminescence spectra of the $\mathrm{ZnO}$ nanorods grown using room temperature and high temperature deposited seed layers were shown in the Fig. 7. The exciton recombination emission (fluorescence) in $\mathrm{ZnO}$ causes the background in the Raman spectra [24], from this observation the nanorods grown at $0.05 \mathrm{M}$ concentration having high luminescence property is confirmed and the photoluminescence characterization was done for those samples. The photoluminescence count of nanorods grown on high temperature deposited seed was higher than the nanorods grown on room temperature deposited seed. This may be due to the nanorods grown at high temperature deposited seed are more crystalline and defect free.

\section{Conclusion}

Vertically aligned $\mathrm{ZnO}$ nanorods were going using hydrothermal method on $\mathrm{ZnO}$ seed layer deposited at room temperature and $250^{\circ} \mathrm{C}$. The molar concentration of chemicals used in hydrothermal process were maintained at 0.05 and $0.1 \mathrm{M}$ and process was carried out for $3 \mathrm{~h}$ and $6 \mathrm{~h}$ respectively. The dispersion of the nanorods was found to be dependent on molar concentration. $A_{1}$ (LO) mode and it overtones were observed in resonant Raman spectra and their count increased with the increase of order of overtone which is a sign of good crystallinity. The photoluminescence count of nanorods grown on high temperature deposited seed was higher than the nanorods grown on room temperature deposited seed.

Acknowledgement: Authors thank Dr. S. Dhara, IGCAR for providing Raman and Photoluminescence characterization facilities.

\section{References}

[1] C. Klingshirn. Zinc oxide. 1st edn (Springer, Heidelberg, 2010).

[2] Yi Sun, Qing Li. CJLCD, 31, 635 (2016).

[3] R. Zhu, R. Yang. Nanotechnology, 25, 345702 (2014).
[4] S. Lee, L. Lee, Y. Chang. Adv. Mater. Res., 1101, 164 (2015).

[5] H. Huang, S. Wang. Angew. Chem., 127, 979 (2014).

[6] V. Suresh, M. Huang, M. Srinivasan, S. Krishnamoorthy. J. Mater. Chem., 22, 21871 (2012).

[7] S. Anas, S. Rahul, K. Babitha, R. Mangalaraja, S. Ananthakumar. Appl. Surf. Sci., 355, 98 (2015).

[8] A. Khan, M. Abbasi, J. Wissting, O. Nur, M. Willander. Phys. St. Sol. Rapid Res. Lett., 7, 980 (2013).

[9] A. Rayerfrancis, Bhargav P. Balaji , N. Ahmed, B. Chandra, S. Dhara. Physica B, 457, 96 (2015).

[10] W. Gao, Z. Li. Int. J. Nanotechn., 6, 245 (2009).

[11] T. Hwang, I. Yang, O. Kwon, M. Ryu, C. Byun, C. Hwang et al. Jpn. J. Appl. Phys., 50: 03CB06 (2011).

[12] P. Thiyagarajan, M. Kottaisamy, N. Rama, Rao M. Ramachandra. Scripta Mater., 59, 722 (2008).

[13] A. Rayerfrancis, Bhargav P. Balaji, N. Ahmed, S. Bhattacharya, B. Chandra, S. Dhara. Solar Cells. Silicon, 9, 31 (2015).

[14] M. Soomro, O. Nur, M. Willander. MRS Proc., 1556 (2013).

[15] T. Goto, S. Yin, T. Sato, T. Tanaka. Int. J. Nanotechn. 10, 48 (2013).

[16] H. Mirzaei, M. Darroudi. Ceram. Int., 43, 907 (2016).

[17] C. Jagadish, S. Pearton. Zinc oxide bulk, thin films and nanostructures (Elsevier, Amsterdam, 2006).

[18] D. Polsongkram, P. Chamninok, S. Pukird, L. Chow, P. Lupan, G. Chai et al. Physica B, 403, 3713 (2008).

[19] J. Venables, G. Spiller, M. Hanbucken. Rep. Prog. Phys., 47, 399 (1984).

[20] K. Kim, H. Kim, C. Lee. Mater. Sci. Eng. B, 98, 135 (2003).

[21] K. Alim, V. Fonoberov, M. Shamsa, A. Balandin. J. Appl. Phys., 97, 124313 (2005).

[22] K. Alim, V. Fonoberov, A. Balandin. Appl. Phys. Lett., 86, 053103 (2005).

[23] Y. Tong, L. Dong, Y. Liu, D. Zhao, J. Zhang, Y. Lu et al. Mater. Lett., 61, 3578 (2007).

[24] Z. Mao, W. Song, X. Xue, W. Ji, L. Chen, J. Lombardi et al. J. Phys. Chem. C, 116, 26908 (2012).

[25] R. Zhang, P. Yin, N. Wang, L. Guo. Solid State Sci., 11, 865 (2009).

[26] S. Chakraborty, S. Dhara, T. Ravindran, S. Pal, M. Kamruddin, A. Tyagi. AIP Advances, 1, 032135 (2011).

Pедактор K.V. Emtsev 